\title{
Treating glioblastoma multiforme with selective high-dose liposomal doxorubicin chemotherapy induced by repeated focused ultrasound
}

This article was published in the following Dove Press journal:

International Journal of Nanomedicine

20 February 2012

Number of times this article has been viewed

\author{
Feng-Yi Yang' \\ Ming-Che Teng' \\ Maggie $\mathrm{Lu}^{2}$ \\ Hsiang-Fa Liang ${ }^{2}$ \\ Yan-Ru Lee' \\ Chueh-Chuan Yen ${ }^{3}$ \\ Muh-Lii Liang ${ }^{4,5}$ \\ Tai-Tong Wong 5
}

'Department of Biomedical Imaging and Radiological Sciences, National Yang-Ming University, Taipei, ${ }^{2}$ Drug Delivery Laboratory, Biomedical Technology and Device Research Laboratories, Industrial Technology

Research Institute, Hsinchu, ${ }^{3}$ Division of Hematology and Oncology, Department of Medicine, Taipei Veterans General Hospital and National Yang-Ming University School of Medicine, Taipei, ${ }^{4}$ Institute of Clinical Medicine, National YangMing University School of Medicine, Taipei, ${ }^{5}$ Department of Neurosurgery, Neurological Institute, Taipei Veterans General Hospital, Taipei, Taiwan

Correspondence: Feng-Yi Yang Department of Biomedical Imaging and Radiological Sciences, School of Biomedical Science and Engineering, National Yang-Ming University,

No 155, Sec 2, Li-Nong St,

Taipei I I22I, Taiwan

Tel +88 622826728 I

Fax +88 6228201095

Email fyyang@ym.edu.tw

Tai-Tong Wong

Department of Neurosurgery,

Neurological Institute, Taipei Veterans

General Hospital

No. 210 , Sec. 2, Shih-Pai Road, Taipei

II 217, Taiwan

Tel $+8862287 \mid 2121$

Fax +88 6228757587

Email ttwong@vghtpe.gov.tw
Background: High-dose tissue-specific delivery of therapeutic agents would be a valuable clinical strategy. We have previously shown that repeated transcranial focused ultrasound is able to increase the delivery of Evans blue significantly into brain tissue. The present study shows that repeated pulsed high-intensity focused ultrasound (HIFU) can be used to deliver high-dose atherosclerotic plaque-specific peptide-1 (AP-1)-conjugated liposomes selectively to brain tumors.

Methods: Firefly luciferase (Fluc)-labeled human GBM8401 glioma cells were implanted into NOD-scid mice. AP-1-conjugated liposomal doxorubicin or liposomal doxorubicin alone was administered followed by pulsed HIFU and the doxorubicin concentration in the treated brains quantified by fluorometer. Growth of the labeled glioma cells was monitored through noninvasive bioluminescence imaging and finally the brain tissue was histologically examined after sacrifice.

Results: Compared with the control group, the animals treated with $5 \mathrm{mg} / \mathrm{kg}$ injections of AP-1 liposomal doxorubicin or untargeted liposomal doxorubicin followed by repeated pulsed HIFU not only showed significantly enhanced accumulation of drug at the sonicated tumor site but also a significantly elevated tumor-to-normal brain drug ratio $(P<0.001)$. Combining repeated pulsed HIFU with AP-1 liposomal doxorubicin or untargeted liposomal doxorubicin has similar antitumor effects.

Conclusion: This study demonstrates that targeted or untargeted liposomal doxorubicin, followed by repeated pulsed HIFU, is a promising high-dose chemotherapy method that allows the desired brain tumor region to be targeted specifically.

Keywords: repeated focused ultrasound, interleukin-4 receptor, blood-brain barrier, brain tumor, target drug delivery

\section{Introduction}

Malignant glioma remains one of the most deadly types of tumor in humans, and glioblastoma multiforme is one of the most common forms of glioma. It is difficult to treat gliomas completely by surgical resection, and therefore radiotherapy and chemotherapy are used to remove residual microscopic tumor material. ${ }^{1}$ Radiotherapy can induce developmental delays, so chemotherapy is recommended as the first line of treatment for children. Presently, chemotherapies are ineffective because many drugs do not reach therapeutic levels in the tumor tissue, either due to limited lipid solubility or to an inability to penetrate an intact blood-brain barrier. ${ }^{2}$ Over the last decade, high-dose chemotherapy has been extensively investigated as first-line therapy in patients having brain tumors with a poor prognosis. Previous studies have demonstrated that first-line, high-dose chemotherapy may 
have a potential survival benefit compared with historical controls treated with standard-dose therapy. ${ }^{3,4}$

It has been reported that human brain tumor cell lines express high levels of plasma membrane interleukin-4 receptors. ${ }^{5}$ Moreover, human brain tumors in situ overexpress interleukin-4 receptors compared with normal brain tissue. ${ }^{6}$ In an immunodeficient xenograft model of human glioblastoma multiforme, an interleukin-4 receptor-targeted cytotoxin has been shown to have a remarkable antitumor effect. ${ }^{7}$ These findings show that therapeutic agents that bind to interleukin-4 receptors may be a useful approach to tumor treatment. ${ }^{8}$ Our previous work has demonstrated that the concentration of Evans blue in tumors and the tumorto-normal brain ratio of Evans blue in the brain is elevated after blood-brain barrier disruption induced by pulsed-high intensity focused ultrasound (HIFU) in the presence of microbubbles. In these circumstances, repeated pulsed HIFU exposure is able to increase further the efficiency of Evans blue delivery into the brain. ${ }^{9-12}$ Another study has shown that magnetic resonance imaging (MRI)-guided focused ultrasound is able to achieve therapeutic levels of liposomal doxorubicin in the brain. ${ }^{13}$ It has been pointed out in other studies that doxorubicin has potential when used clinically against both primary and metastatic brain tumors and that there is improved survival of glioma patients treated by direct intratumoral infusion of doxorubicin; furthermore, doxorubicin is useful against multiple tumor types. ${ }^{14,15}$ However, the concentration of chemotherapeutics required to achieve clinically effective cytotoxicity in tumors is limited by tissue toxicity and by the physiological barriers that prevent the delivery of drugs to the tumor. ${ }^{16}$

Glioblastoma multiforme can be highly vascularized with a leaky vasculature, and thus may be amenable to liposome-based drug delivery systems that lead to enhanced drug deposition while limiting systemic drug exposure. ${ }^{17}$ Receptor-targeted liposomal doxorubicin has been found to be effective in targeting glioma tumors in a brain tumor model. ${ }^{18,19}$ Here, we designed a ligand from atherosclerotic plaque-specific peptide-1 (AP-1) selected from phage display libraries and can locate atherosclerotic plaque tissue and bind to the interleukin-4 receptor because it has the same binding motif to the interleukin-4 protein. ${ }^{8}$ Specifically, in order to allow more specific and efficient delivery of liposomal doxorubicin to brain tumors, an AP-1 actively targeted liposomal antitumor drug specific for interleukin-4 receptors, which is present on the cell membrane of malignant tumors, has been developed. Previous work has demonstrated that AP-1-labeled nanoparticles can be used for targeted drug delivery to tumor tissue. ${ }^{20,21}$ In this study, the integration of AP-1 liposomal doxorubicin and repeated pulsed HIFU was used to deliver and concentrate this high-dose chemotherapeutic agent in brain tumors by the usual systemic dosage. Our aim was to investigate the efficacy of this combined technology using an intracranial brain tumor model.

\section{Materials and methods Preparation of AP-I-labeled liposomal doxorubicin}

Liposomal doxorubicin was prepared using a solvent injection method plus remote loading procedure. Briefly, hydrogenated soybean L- $\alpha$-phosphatidylcholine $(95.8 \mathrm{mg}$, Avanti Polar Lipids Inc, Alabaster, AL), cholesterol (31.9 mg, Sigma-Aldrich, St Louis, MO) and 1,2-distearoyl-sn-glycero3-phosphoethanolamine-N-[methoxy(polyethylene glycol)2000] (DSPE-PEG 2000, 31.9 mg, Avanti Polar Lipids) were dissolved and well mixed in $1 \mathrm{~mL}$ absolute ethanol at $60^{\circ} \mathrm{C}$. The lipid containing ethanol was then injected into a $9 \mathrm{~mL}$ solution of $250 \mathrm{mM}$ ammonium sulfate and stirred for one hour at $60^{\circ} \mathrm{C}$. The mixture was then extruded five times through polycarbonate membranes, each of different pore size (Isopore Membrane Filter, Millipore, Billerica, MA; 0.4, 0.2, 0.1, and $0.05 \mu \mathrm{m}$ ) consecutively in that order at $60^{\circ} \mathrm{C}$ using high-pressure extrusion equipment (Lipex Biomembranes, Vancouver, BC); this produced smaller and smaller sized liposomes.

The liposome suspension was then dialyzed five times against large amounts of $10 \%$ sucrose containing $5 \mathrm{mM}$ $\mathrm{NaCl}$ to remove free ammonium sulfate and ethanol. After dialysis, the liposome suspension was placed in a $50 \mathrm{~mL}$ glass bottle in $60^{\circ} \mathrm{C}$ water bath and was mixed with doxorubicin in a drug to lipid ratio of $1 / 9(\mathrm{w} / \mathrm{w})$, with a final doxorubicin concentration of $2 \mathrm{mg} / \mathrm{mL}$ in a $10 \%$ sucrose solution. The bottle was intermittently shaken in the water bath for one hour at $60^{\circ} \mathrm{C}$ and then cooled down to $4^{\circ} \mathrm{C}$ immediately. The final product was the liposomal doxorubicin.

Due to the presence of a thiol group on each cysteine of the AP-1 peptide (CRKRLDRNC), it is possible to couple AP-1 to liposomes by the thiol-maleimide reaction. Briefly, AP-1 peptide was conjugated to 1,2-distearoyl-sn-glycero3-phosphoethanolamine-N-[maleimide(polyethylene glycol)-2000] (DSPE-PEG2000-MAL, Avanti Polar Lipids) by adding AP-1 to a DSPE-PEG2000-MAL micelle solution at a 2:1 molar ratio, while still mixing at $4^{\circ} \mathrm{C}$ overnight. The free thiol groups were measured with 5,5'-dithiobis-(2-nitrobenzoic acid, Ellman's reagent, Sigma-Aldrich) at an ultraviolet wavelength of $420 \mathrm{~nm}$, 
which confirmed that most of the AP-1 was conjugated with the DSPE-PEG2000-MAL. The AP-1-conjugated DSPEPEG2000 was transferred into the preformed liposomal doxorubicin at a $1.5 \%$ molar ratio of total lipid components and incubated at $60^{\circ} \mathrm{C}$ for one hour to obtain the AP-1 liposomal doxorubicin (Figure 1A). The composition and properties of each preparation are given in Table 1.

\section{Intracranial glioma xenograft model}

Male 6-8-week-old NOD-scid mice were anesthetized by intraperitoneal administration of pentobarbital at a dose of $40 \mathrm{mg} / \mathrm{kg}$ bodyweight. All procedures were performed according to ethical guidelines approved by our Animal Care and Use Committee. The mice were shaved on the head above the nape of the neck, scrubbed with betadine/alcohol, and immobilized in a Cunningham mouse/neonatal rat adaptor stereotactic apparatus (Stoelting, Wood Dale, IL). A $5 \mathrm{~mm}$ skin incision was made along the sagittal suture line and a burr hole drilled into the skull. Malignant human brain glioma cells (GBM8401) were transformed with the luciferase gene (GBM8401-luc), and $2 \times 10^{5}$ GBM8401-luc cells in $2 \mu \mathrm{L}$ culture medium were then injected into the brains of the mice. The glioma cells were stereotactically injected into a single location in the left hemisphere $(0.14 \mathrm{~mm}$ anterior and $2.0 \mathrm{~mm}$ lateral to the bregma) of each mouse at a depth of $3.5 \mathrm{~mm}$ from the brain surface. Next, the burr holes in the skull were sealed with bone wax and the wound was flushed with iodinated alcohol. Bioluminescence quantification of the tumor cells and the established tumor was determined by bioluminescence imaging.

\section{Flow cytometric analysis}

For detection of cell surface interleukin-4 receptor expression on GBM8401-luc cells, the cells were fixed by $1.5 \%$ paraformaldehyde and then stained with anti-interleukin-4 receptor monoclonal antibody (Sf21-derived rhIL-4R; R\&D

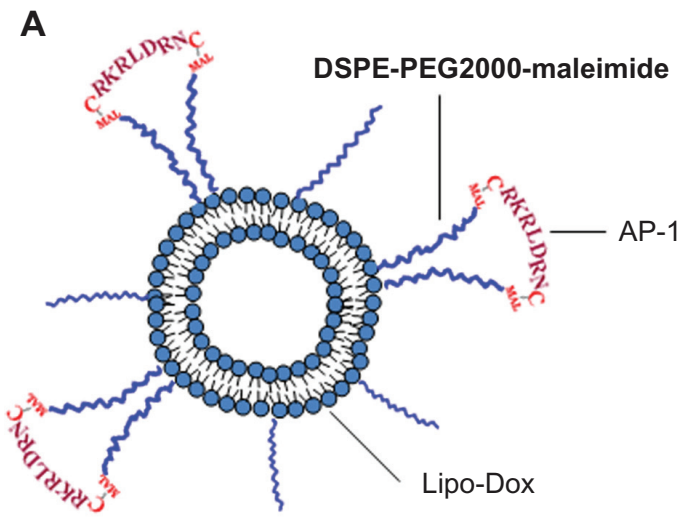

B

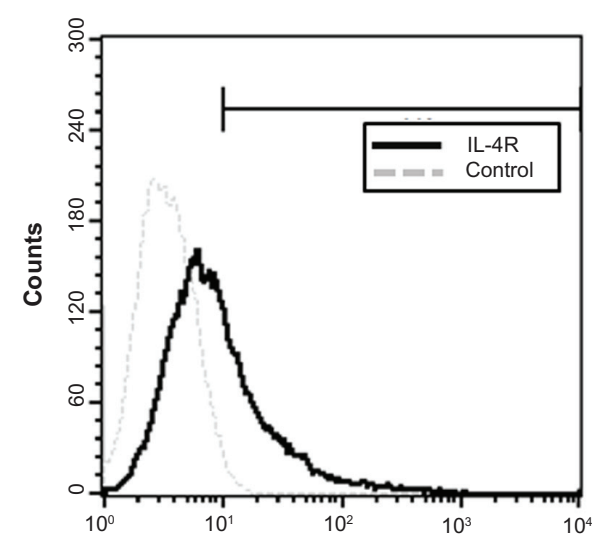

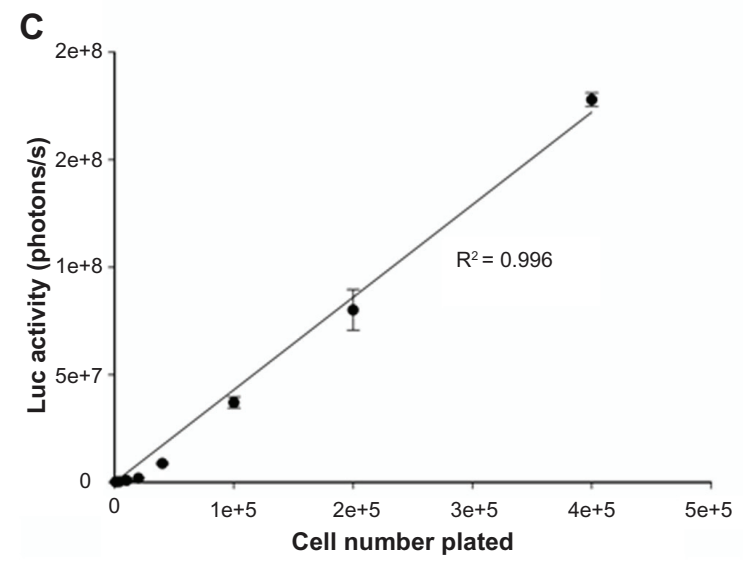

D

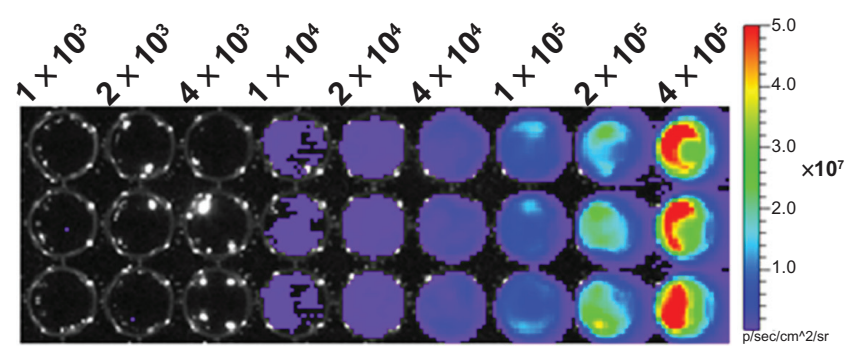

Figure I (A) Schematic representation of the AP-I-conjugated liposome. Liposomes were prepared containing maleimide functional polyethylene glycol chains. Maleimide was used to attach the AP-I peptide through the thiol group on a cysteine. (B) Flow cytometric detection of the cell-surface interleukin-4 receptor on cloned human GBM840I-luc cells. (C) Linearity of measured bioluminescence versus GBM840I-luc cell number. GBM840I-luc cells were plated into 96-well dishes in triplicate in various numbers, and were then imaged by the in vivo imaging system. A strong correlation $\left(R^{2}=0.996\right)$ was observed between luciferase activity and cell numbers. $(D) A$ luciferase image of the plated GBM840 I-luc cells.

Abbreviations: IL-4R, interleukin-4 receptor; Lipo-Dox, liposomal doxorubicin. 
Table I Composition and properties of prepared liposomal doxorubicin and AP-I liposomal doxorubicin

\begin{tabular}{lllll}
\hline Formulation & Composition (molar ratio) & Particle size $(\mathbf{n m})$ & Zeta potential $(\mathrm{mV})$ & Dox concentration $(\mathrm{mg} / \mathrm{mL})$ \\
\hline Lipo-Dox & $6: 4: 0.5$ & $101.0 \pm 24.5$ & $-23.9 \pm 4.8$ & 1.87 \\
AP-I Lipo-Dox & $6: 4: 0.5: 0.15$ & $116.1 \pm 30.3$ & $-26.6 \pm 3.7$ & 1.66 \\
\hline
\end{tabular}

Abbreviation: Lipo-Dox, liposomal doxorubicin.

Systems, Minneapolis, MN) and DyLight488 fluorescentlabeled rabbit antimouse antibody (Rockland Immunochemicals, Gilbertsville, PA). The expression level of the interleukin-4 receptor chain was analyzed by FACSCalibur (Becton Dickinson, San Jose, CA, Figure 1B).

\section{Enzyme-linked immunosorbent assay}

Proteins for enzyme-linked immunosorbent assay (ELISA) analysis were isolated from fresh tissue using T-Per extraction reagent (Pierce Biotechnology Inc, Rockford, IL) according to the manufacturer's recommendations, with the addition of the Halt protease inhibitor cocktail (Pierce Biotechnology Inc). Protein concentrations were determined using the Pierce $660 \mathrm{~nm}$ protein assay reagent (Pierce Biotechnology Inc). Protein levels in the tissue supernatant were measured by ELISA using either a mVEGF Quantikine (R\&D Systems) kit or an ELISA kit for the human interleukin-4 receptor (Uscn Life Science Inc), following the manufacturer's instructions. The results were corrected for total protein concentration and are reported as picograms of vascular endothelial growth factor/interleukin-4 receptor per microgram of protein.

\section{Pulsed HIFU system and treatment}

The pulsed HIFU sonications were generated by a $1.0 \mathrm{mHz}$ single-element focused transducer (A392S, Panametrics, Waltham, MA). The focal zone of the therapeutic transducer was in the shape of an elongated ellipsoid, with a radial diameter $(-6 \mathrm{~dB})$ of $3 \mathrm{~mm}$ and an axial length $(-6 \mathrm{~dB})$ of $26 \mathrm{~mm}$. The ultrasound-driving system and equipment setup were the same as used in our previous studies. ${ }^{22}$ Ultrasound contrast agent (SonoVue, Bracco International, Amsterdam, Netherlands) was injected into the tail vein of the mice about 10 seconds before each sonication. The agent contains phospholipid-coated microbubbles at a concentration of 1 to $5 \times 10^{8}$ bubbles $/ \mathrm{mL}$, with the bubbles having a mean diameter of $2.5 \mu \mathrm{m}$. The ultrasound beam was delivered to one location in the left brain hemisphere centered on the tumor injection site. The following sonication parameters were used: an acoustic power of $2.86 \mathrm{~W}$ (corresponding to peak-negative pressure of $0.7 \mathrm{MPa}$ ) with an injection of $300 \mu \mathrm{L} / \mathrm{kg}$ ultrasound contrast agent; a pulse repetition frequency of $1 \mathrm{~Hz}$; and a duty cycle of 5\%. Each sonication time was 60 seconds.
In order to increase drug accumulation in the tumor region effectively, all mice were sonicated a second time 20 minutes after the first sonication and injected intravenously with ultrasound contrast agent before each sonication.

Five days after tumor cell implantation, the gliomabearing mice were divided into two groups. One group $(n=8)$ received liposomal doxorubicin followed by repeated pulsed HIFU. Another group $(n=8)$ were treated with AP-1 liposomal doxorubicin followed by repeated pulsed HIFU. The concentration of liposomes that was administered to the mice via tail vein injection corresponded to $5 \mathrm{mg} / \mathrm{kg}$. A control group of six mice were injected with GBM8401 glioma cells but received no treatment.

\section{Quantitative measurement of doxorubicin}

Animals were put into a state of deep anesthesia with an overdose of pentobarbital. The brain was perfused by the transcardiac method with normal saline for 3.5 hours after doxorubicin administration in order to flush out unabsorbed doxorubicin from the cerebral vessels. The site of tumor tissue was harvested along with its contralateral counterpart as a control. Doxorubicin was extracted from the tumor and control tissues by homogenization and refrigeration for 24 hours in 20 volumes of acidified ethanol at $4^{\circ} \mathrm{C}$. Tissues were centrifuged at $16,000 \mathrm{~g}$ for 25 minutes at $4^{\circ} \mathrm{C}$ and the supernatant stored at $-20^{\circ} \mathrm{C}$ until the fluorometric assay. ${ }^{23} \mathrm{The}$ concentration of doxorubicin present was measured using a spectrophotometer (PowerWave 340, BioTek, Winooski, VT; excitation $480 \mathrm{~nm}$ and emission $590 \mathrm{~nm}$ ) and the value was determined by taking the average of at least three fluorometric readings. The doxorubicin present in the tissue samples was quantified using a linear regression standard curve derived from seven concentrations of doxorubicin; the amount of doxorubicin is denoted in absorbance per gram of tissue.

\section{Bioluminescence imaging}

To assess the cultured GBM8401-luc cells, we imaged the luciferase signal by adding phosphate buffer solution or colorless Opti-MEM ${ }^{\circledR}$ medium (Invitrogen) with luciferin at a concentration of $0.15 \mathrm{mg} / \mathrm{mL}$. Cells were imaged 10 minutes after luciferin administration (Figure 1C and D). Tumor 
size was quantified by analysis of their biophotonic images obtained from 5 days to 16 days after tumor implantation. The mice received injections of $4.29 \mathrm{mg}$ per mouse of freshly prepared luciferin substrate suspended in phosphate buffer solution. After induction of anesthesia with isoflurane (1.5 L oxygen $+4 \%$ isoflurane per minute), the mice were imaged using an in vivo imaging system (Xenogen, Palo Alto, CA); this occurred 10 minutes after the intraperitoneal injection of luciferin using a one-minute acquisition time in small binning mode. Luciferase activity was viewed and quantified using Living Image software from Xenogen for a region of interest that encompassed the head of the mouse after anesthesia and administration of luciferin substrate.

\section{Magnetic resonance imaging}

MRI was performed using a 3-T MRI system (TRIO 3-T MRI, Siemens Magnetom, Germany). The mice were anesthetized with isoflurane mixed with oxygen during the imaging procedure. A loop coil (Loop Flex Coil, approximately $4 \mathrm{~cm}$ in diameter) for RF reception was used. Tumor progression was evaluated by means of T2-weighted images obtained on day 12 after tumor implantation. The imaging plane was located across the center of the tumor injection site. Parameters for T2-weighted images were as follows: repetition time/echo time $3500 / 75 \mathrm{msec}$; matrix $125 \times 256$; field of view $25 \times 43 \mathrm{~mm}$; and section thickness $1.0 \mathrm{~mm}$.

\section{Histological examination}

After MRI scanning, the brains of the mice were prepared for histological assessment. Two tumor-bearing mice each from the repeated pulsed HIFU plus AP-1 liposomal doxorubicin group, from the repeated pulsed HIFU plus the liposomal doxorubicin treatment group, and the control group were sacrificed 12 days after tumor implantation for histological assessment. The mouse was perfused with saline and 10\% neutral buffered formalin. Tissue sections from the mouse brains were fixed in paraformaldehyde and the sections stained with hematoxylin and eosin (Thermo-Scientific, Waltham, MA). Photomicrographs of the hematoxylin and eosin-stained tissue sections were obtained using a Mirax Scan digital microscope slide scanner (Carl Zeiss, Mirax 3D Histech) with a Plan-Apochromatic 20/0.8 objective. The serial histology images were annotated using Panoramic Viewer software.

\section{Statistical analysis}

All values are shown as the mean \pm standard error of the mean. Statistical analysis was performed using the unpaired
Student $t$-test. Statistical significance was defined as a $P$ value $\leq 0.05$.

\section{Results \\ Concentration of doxorubicin and intensity of pulsed HIFU}

Figure 2A shows the growth of tumor cells in the control mice with no treatment $(n=3)$. Compared with cell numbers on day 5 after implantation, there was a significant increase in tumor cell numbers on days 12,16 , and 20 . The number of tumor cells showed a significant decrease when the mice received a single dose of liposomal doxorubicin at $10 \mathrm{mg} / \mathrm{kg}$ compared with a dose of $5 \mathrm{mg} / \mathrm{kg}$. In addition, there was no significant difference in the bodyweight change after treatment with these two doses of drug (Figure 2B). In the normal brains, pulsed
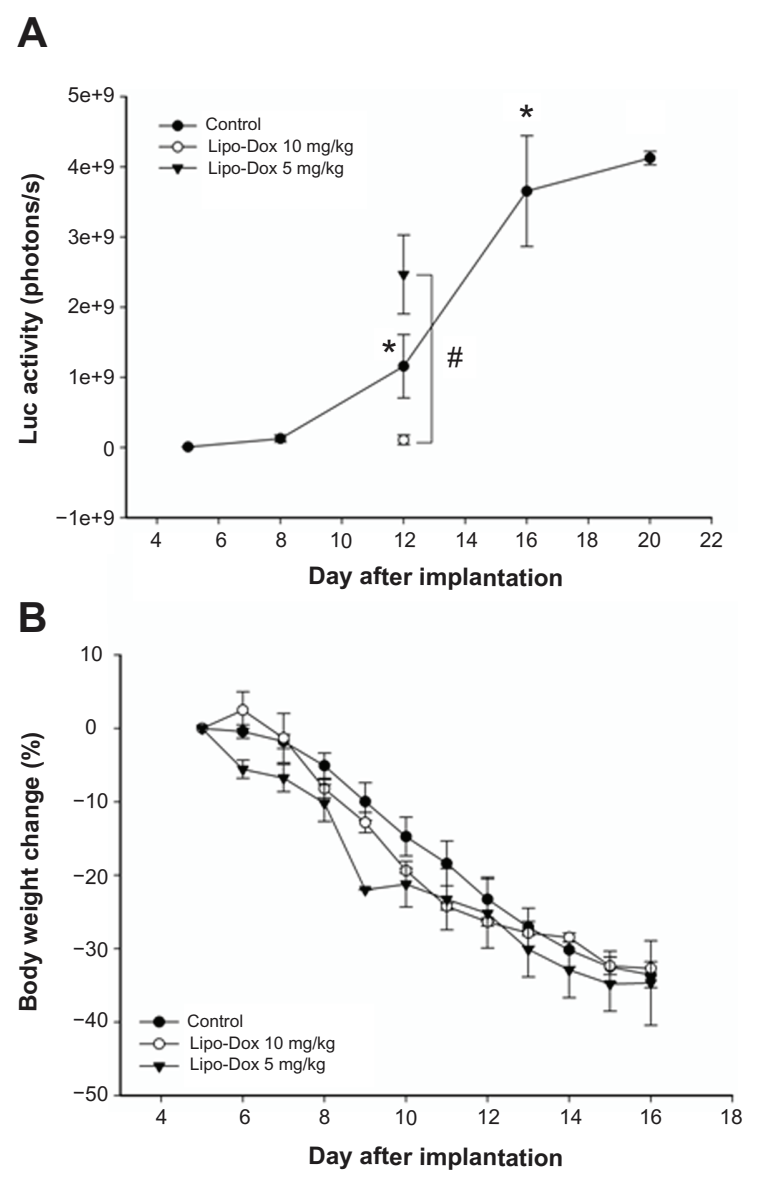

Figure 2 Influence of the concentration of liposomal doxorubicin on tumor progression and bodyweight. (A) Control mice are indicated by $\bullet$. Tumor-bearing mice were treated on day 5 with different doses of liposomal doxorubicin $(\boldsymbol{\nabla}, 5 \mathrm{mg} / \mathrm{kg} ; 0,10 \mathrm{mg} / \mathrm{kg})$. Each point consisted of three mice. *Significant difference in control mice over the three days following cell numbers on day 5 after implantation. There was a significant difference between the tumors treated with liposomal doxorubicin at doses of $5 \mathrm{mg} / \mathrm{kg}$ or $10 \mathrm{mg} / \mathrm{kg}$ and control tumors on day 12 after implantation. (B) Body weight change (relative to day I) of tumor-bearing mice treated with different doses of liposomal doxorubicin.

Notes: $* P<0.05 ;{ }^{*} P<0.05 ; \mathrm{n}=3$.

Abbreviation: Lipo-Dox, liposomal doxorubicin. 
HIFU exposure (2.86 W or $4.29 \mathrm{~W})$ significantly increased the doxorubicin concentration in the brain (Figure 3A). To reduce the systemic toxicity of the drug and tissue damage induced by sonication, doxorubicin accumulation was therefore quantified in tumor-bearing brains after injection of $5 \mathrm{mg}$ / $\mathrm{kg}$ doxorubicin followed by $2.86 \mathrm{~W}$ of repeated sonications. Figure 3B indicates that interleukin-4 receptor expression by the tumor tissue and vascular endothelial growth factor expression by the surrounding tissue did not significantly change after pulsed HIFU sonication of $2.86 \mathrm{~W}$.

\section{Doxorubicin deposition in brains and tumors}

We used spectrophotometry to measure the average tumor doxorubicin concentration (in micrograms per gram of tissue) for three mice from each group. Doxorubicin was extracted from the tumor and contralateral control regions of the harvested brains treated with untargeted liposomal
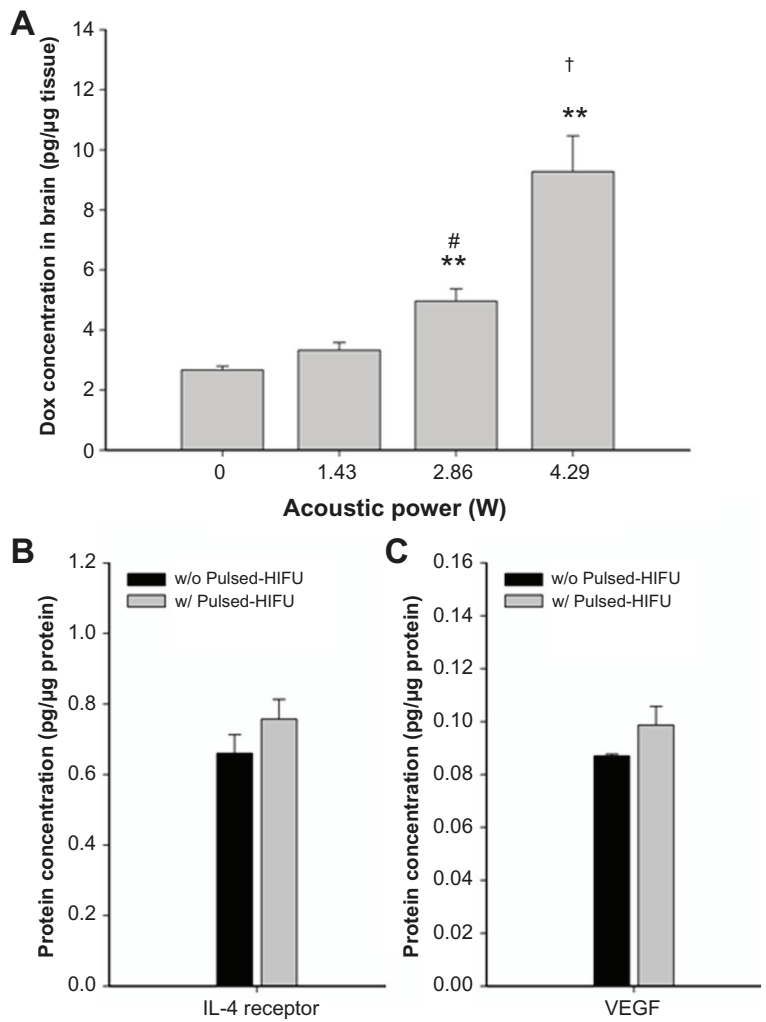

Figure 3 (A) Concentration of doxorubicin delivered to the brain as a function of acoustic power. Compared with the nonsonicated normal brain, there was a significant difference for sonicated brains at $2.86 \mathrm{~W}$ or $4.29 \mathrm{~W}$. ***\#Significant difference compared with the nonsonicated brain and the brain sonicated at $1.43 \mathrm{~W}$, respectively. The concentrations of interleukin-4 receptor protein and vascular endothelial growth factor protein were evaluated in the brain tumor and the neighboring brain, respectively. There was no significant difference in protein expression of the interleukin-4 receptor (B) or vascular endothelial growth factor (C) after pulsed HIFU exposure.

Notes: ${ }^{\#}<0.05$; **P $<0.01$; $\#<<0.01 ; \mathrm{n}=3$ ).

Abbreviations: Lipo-Dox, liposomal doxorubicin; HIFU, high-intensity focused ultrasound; VEGF, vascular endothelial growth factor. doxorubicin or AP-1 liposomal doxorubicin. Figure 4A shows the mean concentration of doxorubicin per unit mass for the brain tumors and the contralateral normal brain tissues with or without repeated sonication after untargeted liposomal doxorubicin or AP-1 liposomal doxorubicin administration. Not only was the concentration of doxorubicin in the nonsonicated tumor significantly greater than that in the contralateral normal brain region, but it was also found that the concentration of doxorubicin significantly increased at the tumor site after repeated sonication compared with the nonsonicated tumor for the two treatments. Repeated pulsed HIFU exposure administered after the drugs were introduced increased the doxorubicin concentration in the tumor by $441 \%$ and $374 \%$ for untargeted liposomal doxorubicin and AP-1 liposomal doxorubicin, respectively. Additionally, the
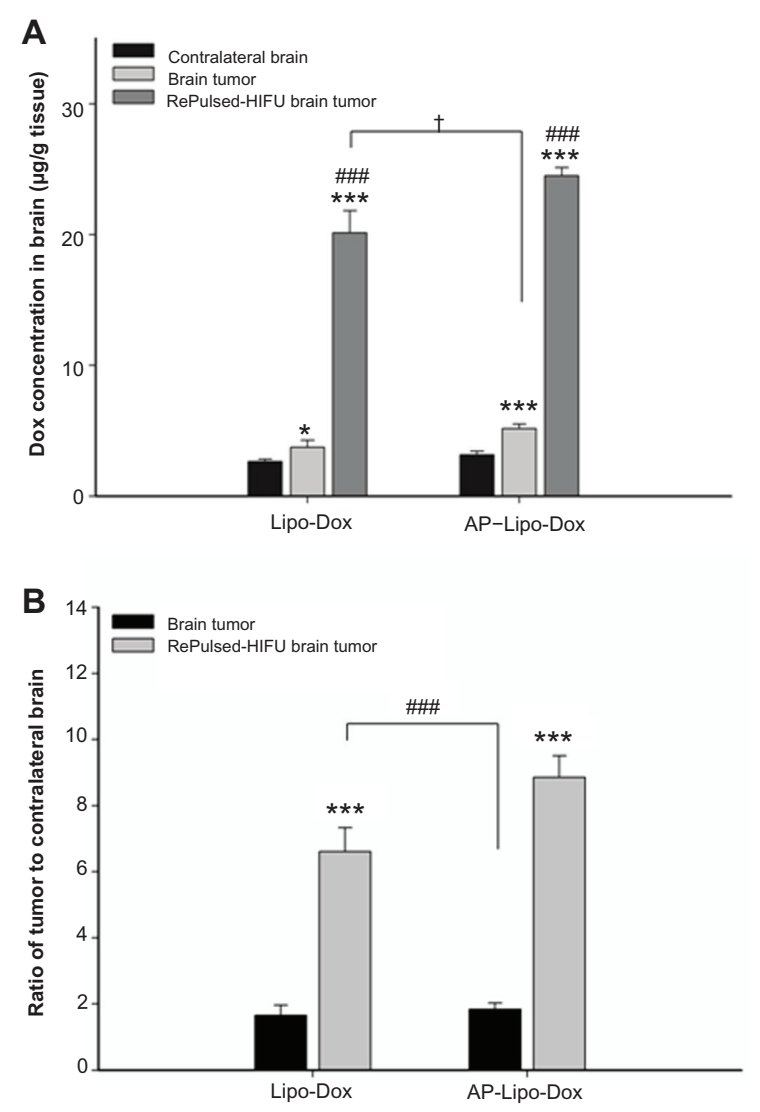

Figure 4 (A) Measurements of untargeted liposomal doxorubicin and AP-I-conjugated liposomal doxorubicin in the contralateral normal brain and the brain tumor without and with repeated sonication. Compared with the contralateral normal brain, there was a significant difference between the control tumors and the repeatedly sonicated tumors with the two drugs. The concentrations of doxorubicin in the brain tumors after repeated sonication were significantly higher than in brain tumors without sonication. ***** Significant difference compared with contralateral normal brain and the nonsonicated brain tumor, respectively. (B) Derived tumor-to-contralateral brain ratios without and with repeated sonication after drug administration. The values of the ratios are significantly elevated after application of repeated sonication.

Notes: $* P<0.05 ; * * * P<0.001 ; \ldots+\cdots<0.001 ; n=3$.

Abbreviations: AP-I, atherosclerotic plaque-specific peptide-I; Lipo-Dox, liposomal doxorubicin; HIFU, high-intensity focused ultrasound. 
concentration of doxorubicin was significantly greater at the tumor site with the untargeted liposomal doxorubicin followed by repeated sonication than for the nonsonicated tumor treated with targeted liposomal doxorubicin without sonication $(P<0.05)$. Compared with the control tumor, there was a significant increase in the derived tumor-tocontralateral brain ratios for the repeatedly sonicated tumor treated with either drug (Figure 4B). Importantly, however, the derived tumor-to-contralateral brain ratio was significantly greater after repeated sonication for the untargeted liposomal doxorubicin group than for the targeted liposomal doxorubicin group without sonication.

\section{Antitumor effect on tumors treated with untargeted or targeted liposomal doxorubicin followed by repeated sonication}

The control tumors and the effect of tumors treated on day 5 by untargeted liposomal doxorubicin or targeted liposomal doxorubicin in combination with repeated pulsed HIFU on tumor progression were monitored by bioluminescence imaging over time (Figure 5). Tumor cells spread rapidly in the untreated control mice (Figure 5, top). When the intracranial brain tumors were treated with untargeted liposomal doxorubicin or targeted liposomal doxorubicin, in both cases followed by repeated pulsed HIFU, a similar pattern of tumor progression was followed. Tumor treatment by liposomal doxorubicin or AP-1 liposomal doxorubicin with repeated sonication significant slowed the growth of the tumors by day 12 after implantation (Figure 6A). Both treatment protocols were associated with no statistically significant decrease in body weight compared with the animals with untreated control tumors (Figure 6B).

\section{MRI and histological evaluation}

The effects of the various treatment protocols on tumor progression were monitored by MRI and also evaluated by hematoxylin and eosin staining on day 12 after implantation (Figure 7). Based on the MRI and histology, tumor progression was found to be consistent with the bioluminescence imaging (Figure 5) and no significant difference in the tumor size was found between the treatment groups.

\section{Discussion}

This study was designed to investigate the applicability of repeated pulsed HIFU exposures when treating brain tumors with high-dose chemotherapeutic agents. This study shows that repeated sonications could significantly increase the concentration of drugs in the brain tumor. Combining the repeated sonications with either untargeted liposomal doxorubicin or targeted liposomal doxorubicin was found to have a similar and significant antitumor effect.

Several studies have indicated that poor distribution and limited penetration by doxorubicin into solid tumors are the main causes of its deficiency as a therapeutic agent. ${ }^{24,25}$ Long-circulating chemotherapies delivered through lipid nanoparticles have been approved to deliver drugs into brain tumors passively by the enhanced permeability and retention effect. ${ }^{26}$

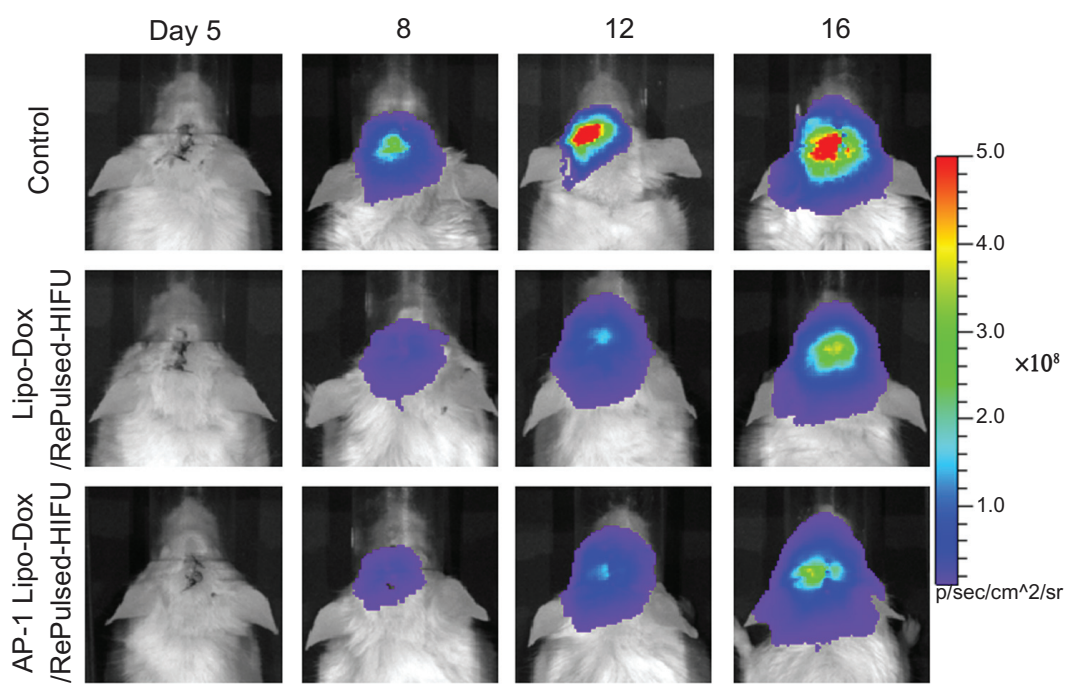

Figure 5 Longitudinal bioluminescence imaging of the brain tumors was monitored from 5 to 16 days after implantation. Firefly luciferase-labeled GBM840 I cells had been implanted into the left forebrain of NOD-scid mice which received no treatment (control), post-treatment with repeated pulsed HIFU after liposomal doxorubicin on day 5 , or post-treatment with repeated pulsed HIFU after AP-I liposomal doxorubicin injection on day 5.

Abbreviations: AP-I, atherosclerotic plaque-specific peptide-I; Lipo-Dox, liposomal doxorubicin; HIFU, high-intensity focused ultrasound. 

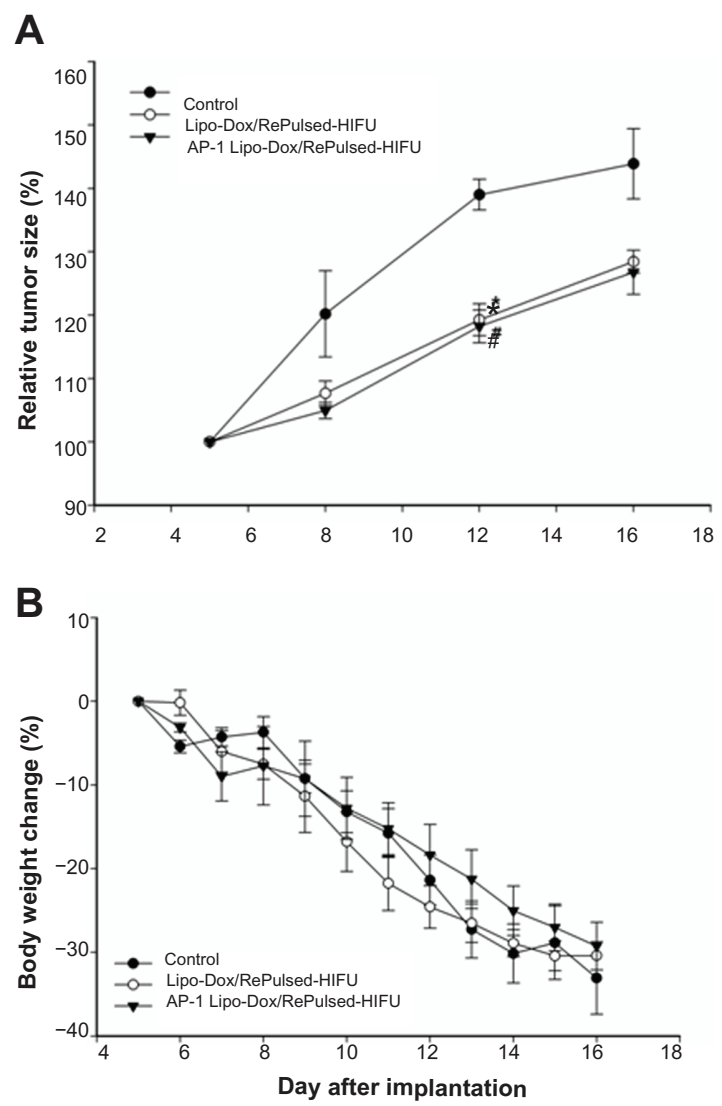

Figure 6 (A) Analysis of increases in tumor size (relative to day 5) is based on data obtained from bioluminescence images in Figure 5. Compared with the control tumor mice, * and ${ }^{*}$ denote a significant difference for untargeted liposomal doxorubicin with repeated sonication and AP-I liposomal doxorubicin with repeated sonication, respectively. (B) Bodyweight change (relative to day 5) of tumor-bearing mice treated by untargeted liposomal doxorubicin with repeated sonication and AP-I liposomal doxorubicin with repeated sonication.

Notes: $* P<0.05 ;{ }^{*} P<0.05$.

Abbreviations: AP-I, atherosclerotic plaque-specific peptide- I; Lipo-Dox, liposomal doxorubicin; HIFU, high-intensity focused ultrasound.

It has been demonstrated that focused ultrasound is able to improve the therapeutic efficacy of liposomal doxorubicin in a breast cancer tumor model. ${ }^{27}$ Previous studies have demonstrated that repeated sonication can significantly increase the efficiency of drug delivery when microbubbles are present for sonication. ${ }^{11,28}$ Our strategy is to use repeated pulsed HIFU to deliver and concentrate the high-dose chemotherapeutic agent into the brain tumor at a minimal systemic dosage (Figure 2A). Moreover, repeated sonications not only significantly enhance the concentration of doxorubicin but also significantly elevate the tumor-to-normal brain ratio in the sonicated tumor tissue.

In our in vitro pilot study, colocalized expression of tumor cells was significantly greater for treatment with AP-1 liposomal doxorubicin than for treatment with untargeted liposomal doxorubicin. Figure 4 shows that repeated pulsed HIFU administered after AP-1 liposomal doxorubicin was introduced gave the highest doxorubicin concentration and

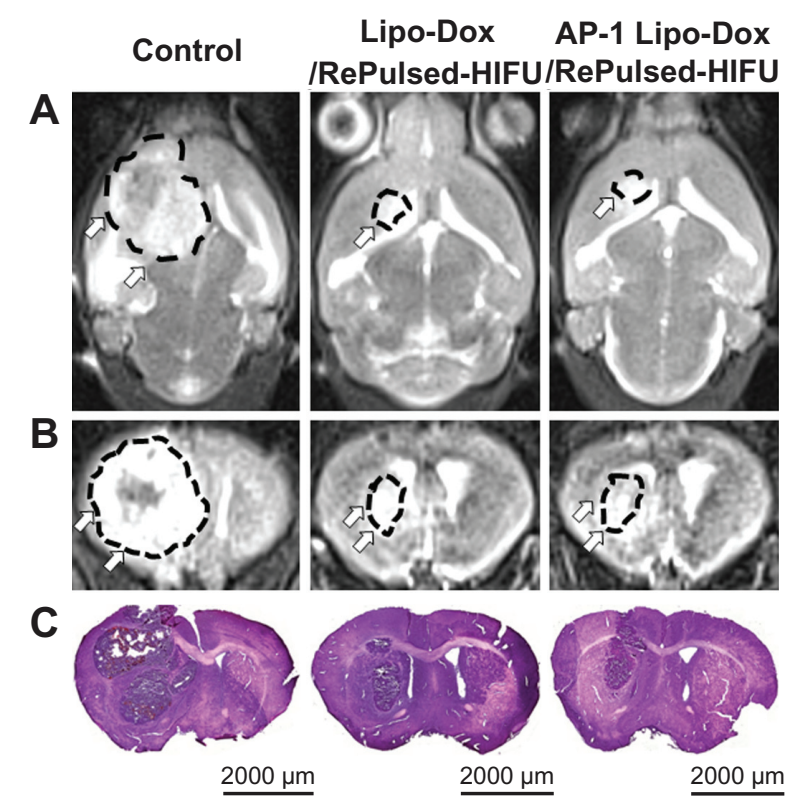

Figure 7 Twelve days after tumor implantation, three mice each from the control group, the untargeted liposomal doxorubicin with repeated sonication group, and the AP-I liposomal doxorubicin with repeated sonication group were imaged by T2-weighted magnetic resonance imaging (A, coronal view; B, axial view), and then sacrificed for hematoxylin and eosin (C) histological examination (scale bar, $2000 \mu \mathrm{m}$ ). Abbreviations: AP-I, atherosclerotic plaque-specific peptide-I; Lipo-Dox, liposomal doxorubicin; HIFU, high-intensity focused ultrasound.

tumor-to-normal brain ratio at the sonicated tumor. However, there were no significant differences between the group treated with AP-1 liposomal doxorubicin and a similar approach using untargeted liposomal doxorubicin. This is consistent with the similar antitumor effects seen in Figure 6A. One possible reason could be that drug delivery was limited by the blood-brain barrier in the in vivo study. Therefore, the targeting ability of AP-1 would not be obvious due to low drug concentration in the tumor tissue. Additionally, the concentration of doxorubicin was significantly greater at the tumor site with the untargeted liposomal doxorubicin followed by repeated sonication than for the nonsonicated tumor treated with targeted liposomal doxorubicin alone $(P<0.05)$. Thus, our results show that doxorubicin accumulation in the tumor occurring with the untargeted drug followed by repeated pulsed HIFU is significantly higher than with targeted doxorubicin alone. In parallel, the tumor-to-normal brain ratio is also significantly elevated by repeated sonication compared with targeted liposomal doxorubicin without sonication (Figure 4B). Therefore, liposomal doxorubicin delivery to the tumor by repeated sonication is much more efficient than that obtained using IL-4 receptortargeted liposomal doxorubicin without sonication.

Chemical modification of drugs and biological agents are methods often used to help an agent cross through the blood-brain barrier into a brain tumor. However, by traditional 
means, in order that sufficient drugs can be delivered to the tumor tissue, the drug dose often needs to be at toxic levels. Pulsed HIFU is a physical assistance method that enhances delivery of chemotherapeutic agents to the targeted region of the brain. There are several mechanisms that may be involved in enhancing drug propagation through the blood-brain barrier, such as blood-brain barrier disruption or widening of the intercellular gaps, both of which can increase transport into the tumor site. Furthermore, pulsed HIFU can shorten peak tumor uptake times and increase peak tumor uptake values compared with unsonicated tumors. ${ }^{29}$ Our previous study has demonstrated that the blood-brain barrier was transiently disrupted by pulsed HIFU. ${ }^{9}$ In the present study, regardless of whether untargeted or targeted drugs are used, focused ultrasound offers a local and efficient method for delivering either drug (Figure 4A). High-dose chemotherapy has yielded favorable results when treating brain metastases or primary brain tumors. ${ }^{30}$ Using pulsed HIFU to allow delivery of high-dose therapeutic agents to brain tumors using standard chemotherapeutic doses is an innovative approach to treatment.

This study demonstrates that repeated pulsed HIFU is able to deliver high-dose chemotherapeutic drugs to brain tumors and improve the antitumor effect of the drugs at minimal systemic dosage. Repeated pulsed HIFU seems to be a good method of achieving local high-dose chemotherapy for malignant glioma or other brain diseases without increasing systemic toxicity.

\section{Acknowledgments}

This study was supported by grants from the National Science Council of Taiwan (100-2321-B-010-010 and 99-2321-B-010-017), Cheng Hsin General Hospital Foundation (100F117CY25), Veterans General Hospital University System of Taiwan Joint Research Program (VGHUST100-G1-3-3 and V100E6-007), Yen Tjing Ling Medical Foundation (CI-100-17), and Department of Health of Taiwan (DOH101TD-PB-111-TM012 and DOH101-TD-C-111-007).

\section{Disclosure}

The authors report no conflicts of interest in this work.

\section{References}

1. Deen DF, Chiarodo A, Grimm EA, et al. Brain tumor working group report on the 9th international conference on brain tumor research and therapy. Organ System Program, National Cancer Institute. J Neurooncol. 1993;16:243-272.

2. Cordon-Cardo C, O'Brien JP, Casals D, et al. Multidrug-resistance gene (P-glycoprotein) is expressed by endothelial cells at blood-brain barrier sites. Proc Natl Acad Sci U S A. 1989;86:695-698.
3. Motzer RJ, Mazumdar M, Gulati SC, et al. Phase II trial of high-dose carboplatin and etoposide with autologous bone marrow transplantation in first-line therapy for patients with poor-risk germ cell tumors. $J$ Natl Cancer Inst. 1993;85:1828-1835.

4. Motzer RJ, Mazumdar M, Bajorin DF, Bosl GJ, Lyn P, Vlamis V. High-dose carboplatin, etoposide, and cyclophosphamide with autologous bone marrow transplantation in first-line therapy for patients with poor-risk germ cell tumors. J Clin Oncol. 1997;15:2546-2552.

5. Puri RK, Leland P, Kreitman RJ, Pastan I. Human neurological cancer cells express interleukin-4 (IL-4) receptors which are targets for the toxic effects of IL4-pseudomonas exotoxin chimeric protein. Int $J$ Cancer. 1994;58:574-581.

6. Joshi BH, Leland P, Asher A, Prayson RA, Varricchio F, Puri RK. In situ expression of interleukin-4 (IL-4) receptors in human brain tumors and cytotoxicity of a recombinant IL-4 cytotoxin in primary glioblastoma cell cultures. Cancer Res. 2001;61:8058-8061.

7. Husain SR, Behari N, Kreitman RJ, Pastan I, Puri RK. Complete regression of established human glioblastoma tumor xenograft by interleukin-4 toxin therapy. Cancer Res. 1998;58:3649-3653.

8. Hong HY, Lee HY, Kwak W, et al. Phage display selection of peptides that home to atherosclerotic plaques: Il-4 receptor as a candidate target in atherosclerosis. J Cell Mol Med. 2008;12:2003-2014.

9. Yang FY, Wang HE, Lin GL, et al. Micro-SPECT/CT-based pharmacokinetic analysis of 99 mtc-diethylenetriaminepentaacetic acid in rats with blood-brain barrier disruption induced by focused ultrasound. J Nucl Med. 2011;52:478-484.

10. Yang FY, Horng SC, Lin YS, Kao YH. Association between contrastenhanced MR images and blood-brain barrier disruption following transcranial focused ultrasound. J Magn Reson Imaging. 2010;32: 593-599.

11. Yang FY, Lin YS, Kang KH, Chao TK. Reversible blood-brain barrier disruption by repeated transcranial focused ultrasound allows enhanced extravasation. J Control Release. 2011;150:111-116.

12. Yang FY, Liu SH, Ho FM, Chang CH. Effect of ultrasound contrast agent dose on the duration of focused-ultrasound-induced blood-brain barrier disruption. J Acoust Soc Am. 2009;126:3344-3349.

13. Treat LH, McDannold N, Vykhodtseva N, Zhang Y, Tam K, Hynynen K. Targeted delivery of doxorubicin to the rat brain at therapeutic levels using MRI-guided focused ultrasound. Int J Cancer. 2007;121:901-907.

14. Walter KA, Tamargo RJ, Olivi A, Burger PC, Brem H. Intratumoral chemotherapy. Neurosurgery. 1995;37:1128-1145.

15. Cummings J, McArdle CS. Studies on the in vivo disposition of adriamycin in human tumours which exhibit different responses to the drug. Br J Cancer. 1986;53:835-838.

16. Floyd JD, Nguyen DT, Lobins RL, Bashir Q, Doll DC, Perry MC. Cardiotoxicity of cancer therapy. J Clin Oncol. 2005;23:7685-7696.

17. Allen TM, Cullis PR. Drug delivery systems: Entering the mainstream. Science. 2004;303:1818-1822.

18. Madhankumar AB, Slagle-Webb B, Wang X, et al. Efficacy of interleukin-13 receptor-targeted liposomal doxorubicin in the intracranial brain tumor model. Mol Cancer Ther. 2009;8:648-654.

19. Madhankumar AB, Slagle-Webb B, Mintz A, Sheehan JM, Connor JR. Interleukin-13 receptor-targeted nanovesicles are a potential therapy for glioblastoma multiforme. Mol Cancer Ther. 2006;5:3162-3169.

20. Kim JH, Bae SM, Na MH, et al. Facilitated intracellular delivery of peptide-guided nanoparticles in tumor tissues. $J$ Control Release. September 16, 2011. [Epub ahead of print.]

21. Wu XL, Kim JH, Koo H, et al. Tumor-targeting peptide conjugated $\mathrm{pH}-$ responsive micelles as a potential drug carrier for cancer therapy. Bioconjug Chem. 2010;21:208-213.

22. Yang FY, Lin GL, Horng SC, et al. Pulsed high-intensity focused ultrasound enhances the relative permeability of the blood-tumor barrier in a glioma-bearing rat model. IEEE Trans Ultrason Ferroelectr Freq Control. 2011;58:964-970.

23. Bachur NR, Moore AL, Bernstein JG, Liu A. Tissue distribution and disposition of daunomycin (ncs-82151) in mice: Fluorometric and isotopic methods. Cancer Chemother Rep. 1970;54:89-94. 
24. Primeau AJ, Rendon A, Hedley D, Lilge L, Tannock IF. The distribution of the anticancer drug doxorubicin in relation to blood vessels in solid tumors. Clin Cancer Res. 2005;11:8782-8788.

25. Lankelma J, Dekker H, Luque FR, et al. Doxorubicin gradients in human breast cancer. Clin Cancer Res. 1999;5:1703-1707.

26. Sharma US, Sharma A, Chau RI, Straubinger RM. Liposome-mediated therapy of intracranial brain tumors in a rat model. Pharm Res. 1997; 14:992-998.

27. Frenkel V, Etherington A, Greene M, et al. Delivery of liposomal doxorubicin (Doxil) in a breast cancer tumor model: Investigation of potential enhancement by pulsed-high intensity focused ultrasound exposure. Acad Radiol. 2006;13:469-479.
28. Bekeredjian R, Chen S, Frenkel PA, Grayburn PA, Shohet RV. Ultrasound-targeted microbubble destruction can repeatedly direct highly specific plasmid expression to the heart. Circulation. 2003;108: 1022-1026.

29. Khaibullina A, Jang BS, Sun H, et al. Pulsed high-intensity focused ultrasound enhances uptake of radiolabeled monoclonal antibody to human epidermoid tumor in nude mice. J Nucl Med. 2008;49:295-302.

30. Kollmannsberger C, Nichols C, Bamberg M, et al. First-line high-dose chemotherapy $+/$ - radiation therapy in patients with metastatic germ-cell cancer and brain metastases. Ann Oncol. 2000;11:553-559.

\section{Publish your work in this journal}

The International Journal of Nanomedicine is an international, peerreviewed journal focusing on the application of nanotechnology in diagnostics, therapeutics, and drug delivery systems throughout the biomedical field. This journal is indexed on PubMed Central, MedLine, CAS, SciSearch ${ }^{\circledR}$, Current Contents ${ }^{\circledR} /$ Clinical Medicine,
Journal Citation Reports/Science Edition, EMBase, Scopus and the Elsevier Bibliographic databases. The manuscript management system is completely online and includes a very quick and fair peer-review system, which is all easy to use. Visit http://www.dovepress.com/ testimonials.php to read real quotes from published authors. 